(C) 2011 IEEE. Personal use of this material is permitted. Permission from IEEE must be obtained for all other uses, in any current or future media, including reprinting/republishing this material for advertising or promotional purposes, creating new collective works, for resale or redistribution to servers or lists, or reuse of any copyrighted component of this work in other works. 


\title{
Quantification Limits of Iterative PET Reconstruction Algorithms and Improved Estimation of Kinetic Constants
}

\author{
E. Herranz, Student Member, IEEE, J. L. Herraiz, Member, IEEE, E, Vicente, S. España, Member, IEEE, \\ M. Desco, J. J. Vaquero, Senior Member, IEEE, J. M. Udías, Member, IEEE
}

\begin{abstract}
Quantification of tracer kinetics is often accomplished from time-activity curves of a region of interest of dynamic PET images. The choice of reconstruction method may affect the timeactivity curves and hence the estimated kinetic parameters. Several studies have shown that statistical-iterative methods, due to non-negativity constrains, may exhibit a quantification bias in low activity regions and thus these methods, in spite of the better image quality they provide, are seldom used to estimate kinetic constants. By means of realistic dynamic simulations, we have investigated the quantitative properties of statistical-iterative (OSEM, both 2D and 3D) and FBP reconstruction methods and the accuracy of the kinetic parameters derived from images reconstructed with each algorithm. We focus on the procedure to fit kinetic constants to data. Our results show that, with appropriate measures to account for quantification bias, iterative reconstructions may be suited to derive kinetic constants from dynamic PET acquisitions.
\end{abstract}

\section{INTRODUCTION}

Quantification of tracer kinetics using dynamic PET provides important information about physiological and biochemical processes in human and animals. In these studies, variation

Manuscript received November 15, 2011. This work was supported from Comunidad de Madrid (ARTEMIS S2009/DPI-1802), Spanish Ministry of Science and Innovation (ENTEPRASE Grant, PSE-300000-2009-5) and PRECISION grant IPT-300000-2010-3, UCM (Grupos UCM, 910059), and european regional funds and CPAN, Centro de Física de Partículas, Astropartículas y Nuclear (CSD-2007-00042@Ingenio2010-12). This study has been (partially) funded by CDTI under the CENIT Programme (AMIT Project). Part of the calculations of this work was performed in the "Clúster de Cálculo de Alta Capacidad para Técnicas Físicas" funded in part by UCM and in part by UE with European regional funds".

E. Herranz, E. Vicente and J. M. Udias are with the Grupo de Física Nuclear, Dpto. Física Atómica, Molecular y Nuclear, Universidad Complutense de Madrid, Spain (telephone: +34-91-394-4484, email: eherranz@nuclear.fis.ucm.es).

J.L. Herraiz was with the Grupo de Física Nuclear, Universidad Complutense de Madrid, Spain. He is now with the Madrid-MIT M+Visión consortium.

S. España was with the Grupo de Física Nuclear, Universidad Complutense de Madrid, Spain. He is now with the Department of Electronics and Information Systems, MEDISIP, Ghent University-IBBT-IBiTech, De Pintelaan 185 block B, B-9000 Ghent, Belgium (S. España e-mail: samuel@nuclear.fis.ucm.es).

J. J. Vaquero and M. Desco are with the Departamento de Bioingeniería e Ingeniería Aeroespacial, Universidad Carlos III de Madrid, Spain and with the Unidad de Medicina y Cirugía Experimental, Hospital General Universitario Gregorio Marañón, Madrid, Spain

M. Desco is also with Unidad de Medicina y Cirugía Experimental, Hospital General Universitario Gregorio Marañón, Madrid, Spain and CIBERSAM, Instituto de Salud Carlos III, Madrid, Spain.

E. Vicente is also with Instituto de Estructura de la Materia, Consejo Superior de Investigaciones Científicas (CSIC), Madrid, Spain. with time of the activity of the radiotracer is obtained acquiring data in consecutive time frames. Either analytic or iterative algorithms may be used to reconstruct the activity map corresponding to each time frame. Thus, the time activity curve (TAC) for a region of interest (ROI) can be extracted from the images and employed to estimate kinetic parameters, based for instance in compartmental models [1]. Different reconstruction methods will exhibit different quantifications errors, which may affect the accuracy of the time activity curve and hence of the kinetic parameters. To date, FBP algorithms are considered better than iterative ones [2] for quantification purposes. Indeed, iterative image reconstruction algorithms may result in bias of the reconstructed activity concentration, especially in cold regions, due to the use of non-negativity constrains. Such bias may lead to errors in the biological parameters derived from dynamic PET images [2]-[3]. However, iterative methods yield better images (increased resolution, better recovery coefficients and larger signal to noise ratios) than FBP, especially for low count acquisitions, as it is usually the case of kinetic studies. In this work, we investigate the quantitative properties of the iterative OSEM algorithm (2D and 3D versions) and of the FBP one, and assess the impact of the choice of reconstruction methods on the kinetic model parameters inferred from the images. We also examine the quantification bias present in OSEMreconstructed low-count simulated PET acquisitions and estimate the errors in the estimates of the biological parameters that this bias may induce. While most authors try to estimate and eliminate this bias, here instead we try to take this bias into consideration during the fit of kinetic parameters.

\section{METHODS}

\section{A. Dynamic simulations}

Realistic simulations of dynamic acquisitions were performed with PeneloPET [4] for the ARGUS small-animal PET scanner (SEDECAL, Madrid, Spain) [5]. A "Dynamic QC-NEMA phantom" of $60 \mathrm{~s}$ per frame was created with two cylindrical regions by varying the FDG activity of these regions within the dynamic rage of the simulated scanner under usual working conditions. These cylinders were surrounded by a uniform region with a constant background activity concentration $(12.4 \mu \mathrm{Ci} / \mathrm{ml})$. One of the cylinders was simulated with no initial activity (cold region) and the other was simulated with an initial activity concentration of four times the concentration of the uniform region (hot region). A 
one-compartmental model with two parameters was used to describe the evolution of activity over time in each cylinder and further simulations at these subsequent activities were performed. Equations (1) and (2) show the kinetic description for the activities of the hot and cold cylinder, respectively.

$$
\begin{gathered}
C_{H}(t)=C_{a} \cdot\left[\frac{k_{1 H}}{k_{2 H}}+\left(4-\frac{k_{1 H}}{k_{2 H}}\right) \cdot e^{-k_{2 H} t}\right] \\
C_{C}(t) \quad C_{a} \cdot \frac{k_{1 C}}{k_{2 C}} \cdot\left[1-e^{-k_{2 C} t}\right]
\end{gathered}
$$

where $\mathrm{C}(\mathrm{t})$ is the time course of FDG in the cylinder (hot or cold), $\mathrm{C}_{\mathrm{a}}$ is the activity concentration in uniform region and $\mathrm{k}_{1}$ and $k_{2}$ are the rate constant of the model $\left(k_{1 H}=0.01 \min ^{1}\right.$, $\mathrm{k}_{2 \mathrm{H}}=0.05 \min ^{1}$, for hot cylinder, and $\mathrm{k}_{1 \mathrm{C}}=0.05 \min ^{1}, \mathrm{k}_{2 \mathrm{C}}=0.01$ $\min ^{1}$, for cold cylinder).

\section{B. Image reconstruction}

Simulated data were reconstructed using FBP with Hanning filter at 0.5 of the Nyquist frequency. For 3D-iterativereconstruccion FIRST (a commercially available code based on 3D-OSEM) was used [6]. The parameters of the reconstruction were chosen so that they yield a similar level of noise in the uniform regions for both FBP, 2D-OSEM and 3DOSEM images.

\section{Introduction of the quantification error}

Systematic error (or bias) in the quantification of ROIs at different activities was studied. Estimates of quantification bias were obtained from ROI measurements in the simulated acquisitions from the dynamical phantom, for which the actual activity values were known. The standard deviation of the activity within the ROI was used as a measure of statistical error of the reconstruction (Fig. 1). The deviations of the reconstructed activity from the real one were computed for each method. As expected [2]-[3], a significant excess of counts (positive bias) at regions of relative low activity was found for iterative methods (Fig. 1).

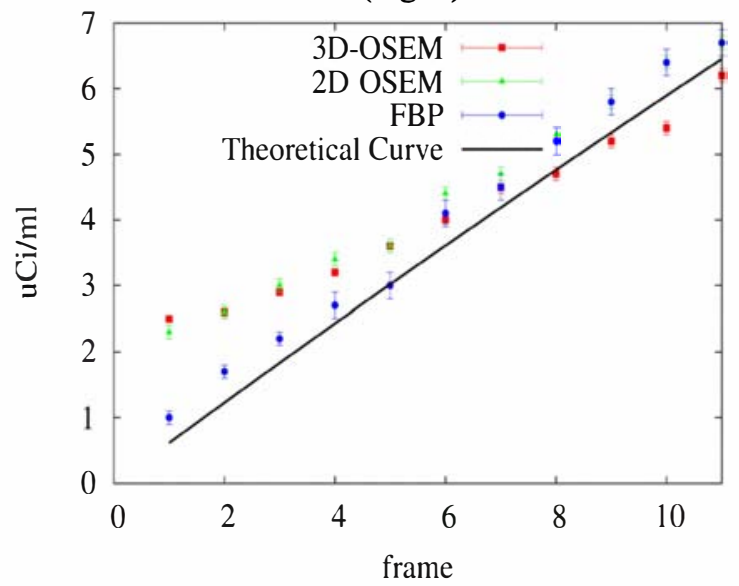

Fig. 1 Comparison of the quantification properties of the three methods of reconstruction, 3D-OSEM, 2D-OSEM and FBP, in a region of the phantom with low activity concentration, cold region. Results are expressed as the average of voxel values within the ROI. Statistical errors are estimated from the ratio of the standard deviation of the activity values and the squared root of the number of voxels in the ROI.
FBP showed better quantification at these low activity regions but with larger statistical uncertainties. Guided by the results of these simulations, we will introduce systematic quantification errors for each reconstruction method. These are $1 \mu \mathrm{Ci} / \mathrm{ml}$ for the FBP algorithm, for the whole activity range studied. For the iterative reconstructions, we will use $2 \mu \mathrm{Ci} / \mathrm{ml}$, for activity concentrations smaller than $3 \mu \mathrm{Ci} / \mathrm{ml}$, and 1 $\mu \mathrm{Ci} / \mathrm{ml}$, for activity concentrations higher than $3 \mu \mathrm{Ci} / \mathrm{ml}$. Time-activity curves were extracted from the reconstructed images using ROIs placed in the hot and cold cylinders.

\section{Estimation of kinetic constant and its range of variation}

Kinetic constants were estimated by fitting kinetic models to the time-activity curves of selected regions, by means of a genetic algorithm, minimizing a chi-square-like function [7]:

$$
\chi^{2} \quad \sum_{i=1}^{N} \frac{\left(Y_{i}-Y\left(X_{i}\right)\right)^{2}}{\left(\Delta Y_{i}\right)^{2}}
$$

where $Y_{i}$ are the activity concentration data and $Y\left(X_{i}\right)$ are the activity concentration estimated by the kinetic model at frame $i$ for a given value of kinetic constants. $\Delta Y_{i}$ represent the estimated errors in the measured activities. Minimizing this quantity would yield estimates of the most likely kinetic parameters. Our goal is to obtain estimations of the kinetic constant and of the ranges of most likely values for these constants, given the uncertainties of the measurements. Thus, a detailed study of uncertainties and their effect on the kinetic constants had to be performed.

If the deviations are normally distributed, and $\Delta Y_{i}$ represent the statistical error, the quantity in Eq. (3) would follow [7] a $\chi^{2}$ distribution with the corresponding degrees of freedom (number of dynamic frames quantized to get a value of the activity at a given time, minus the number of kinetic constants). The likely range of variation of the deduced kinetic constant can be then obtained from a standard confidence level $\chi^{2}$ test. However, for all reconstruction methods, systematic deviations exist, which are not normally distributed and thus the quantity in Eq. (3) will not in general follow a $\chi^{2}$ distribution. We thus derive the likely range of variation of derived kinetic constants generating many pseudo data sets compatible with the true values of activity in the simulated phantoms, if the expected quantification errors of the reconstruction algorithm are taken into account.

For instance, in the case of the iterative reconstructions, any real activity value in the range $0-3 \mu \mathrm{Ci} / \mathrm{ml}$ would result in a measured activity of approximately $3 \mu \mathrm{Ci} / \mathrm{ml}$, due to the quantification error. We thus generate 10,000 random sets of true activities which can correspond to the same observed measurements and for each set we fit the kinetic constants, obtaining a set of 10,000 pairs of kinetic constants compatible with the observed measurements. The dispersion of values of the kinematics constants within these pseudodata sets, yields an indication of the likely range of error in the determination of the kinetic parameters.

We studied whether the constants derived were in agreement with the exact (known) values, within the error bands provided 
by either the simple $\chi^{2}$ test or the pseudodata sets analysis outlined in the previous paragraphs, comparing the results of two variations of Eq. (3) : i) one which only takes into account the (relatively small) statistical uncertainties in the simulated data and, ii) one in which the estimates of the systematic deviations from the real activities for each quantification method, as previously estimated, were included as additional uncertainty in the fitting procedure.

As just said, in order to estimate the variation range of the kinetic constants and to characterize how well the data constrain the model, two tests were carried out: 1) $\chi^{2}$-test: variation range was obtained from confidence regions on the fitted model parameters assessed for a $95 \%$ confidence level region. To determine the confidence regions, we identified the region in parameter space where the $\chi^{2}$ in Eq. (3) is less than the value given by the confidence level and found in the standard tables. In our study, for a $95 \%$ confidence level, this value is around 1.3 per degree of freedom, for the 34 degrees of freedom of the cases studied. The likely region where the real values of the fitted parameters are supposed to be, will be given by the region for which the $\chi^{2}$ value remains below 1.3 (Fig.2). 2) Pseudodata analysis: we generated 10,000 sets of randomly distributed time-activity curves compatible, taking into account the systematic (uniformly distributed) and statistical (normally distributed) quantification errors previously discussed, with the actual measurements taken from the images. For each set of data, we obtain the best fit parameters $k_{1}$ and $k_{2}$ by minimization of the quantity in Eq. (3), with the denominator including only statistical uncertainties (i), on one hand, and when they also included systematic errors added in quadrature (ii).

\section{RESULTS}

Fig. 2 (top) shows the two dimensional map of $\chi^{2}$ of Eq. (3) as a function of $\mathrm{k}_{1 \mathrm{H}}$ and $\mathrm{k}_{2 \mathrm{H}}$ parameters for the hot region of the simulated phantom, 3D-OSEM reconstruction algorithm, variation range is obtained with the procedure mentioned in 1), that is, from tables of $\chi^{2}$ distributions. One problem with this procedure is that, as seen in Fig. 1, the deviations from the activities measured on the images and the real activity values are much larger than the statistical uncertainties. This in turns yields a very high value of the $\chi^{2}$ in all cases, much larger than the $95 \%$ confidence level value. The standard procedure in this case [7] consists in rescaling the 'statistical' errors by the needed factor until obtaining reasonable values of $\chi^{2}$. Once this rescaling is made, the range of variation, for instance $\Delta \mathrm{k}_{1}$, can be read from the confidence ellipse. For the case ii), when the systematic errors are included in the denominator of Eq. (3), the values of $\chi^{2}$ are within the normal bounds of a good fit and thus no rescaling of the $\chi^{2}$ values is needed.

In Fig. 2 we also show the usual choice for the estimation of the kinetic parameter error, that is, the vertical error bar represents the estimation of the expected error in $k_{2 H}$ for the optimal value of $\mathrm{k}_{1 \mathrm{H}}$ (central point of the confidence ellipse). Notice that, as both kinetic constants are strongly correlated, given independent uncertainty intervals for both parameters is an oversimplification that may just serve as a raw estimate of errors.

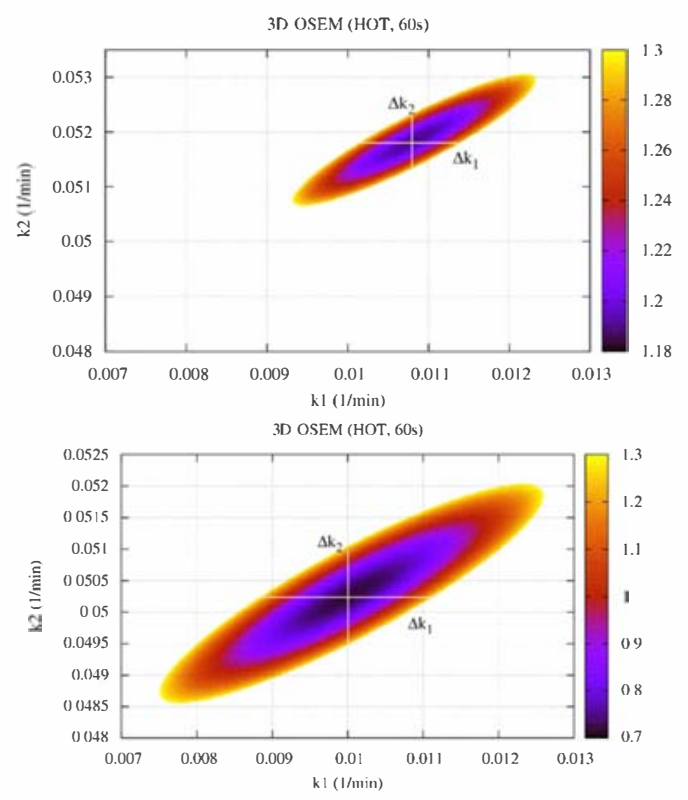

Fig. 2. Confidence regions obtained from test $\chi^{2}$ for hot region of the simulated phantom for 3D-OSEM reconstruction algorithm for case i) (top) and ii) (bottom). Values are given divided by degree of freedom.

Fig. 3 shows the two dimensional map of $\chi^{2}$ as a function of $\mathrm{k}_{1 \mathrm{H}}$ and $\mathrm{k}_{2 \mathrm{H}}$ for the hot region of the simulated phantom for 3D-OSEM reconstruction algorithm when the variation range is obtained from the analysis of 10,000 pseudodata sets previously described. This procedure should be considered as the gold standard to obtain the range of variation of the kinetic constants, as it includes realistically the effect of both statistical and systematic uncertainties on each measurement.

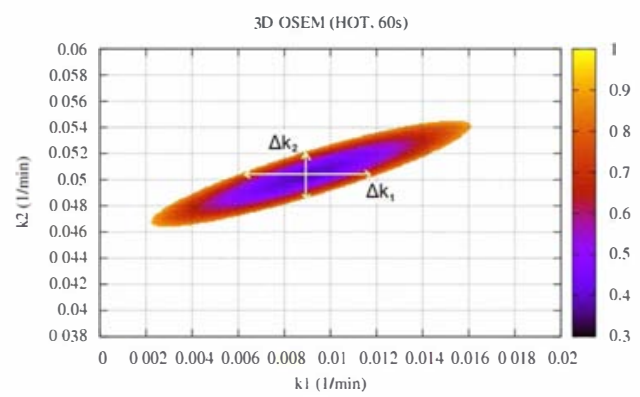

Fig. 3. Confidence ellipse obtained from pseudodata analysis of the hot region of the simulated phantom with 3D-OSEM reconstruction algorithm. The region which includes $95 \%$ of the 10,000 generated pseudodata sets is used to define the likely rang of variation of the constants. Values of the $\chi^{2}$ quantity of EQ.(3), per degree of freedom, are plotted.

\section{A. Accuracy of the quantification}

In Fig. 1, the TACs of the hot and cold regions extracted from 2D-OSEM, 3D-OSEM and FBP images are compared with the theoretical values. We found, as it is usually acknowledged [2,3] that FBP images have the least relative quantification errors in cold regions, where iterative OSEM 
images show activity in excess, presumably due to the nonnegativity constrains [3]. These systematic deviation, or bias, in the low activity region, are by far larger than the statistical errors estimated as mentioned in Fig. 3, for the simulations we made that we note are very similar to the conditions of real studies. For relatively high activity concentrations, both OSEM and FBP exhibit similar accuracy of quantification.

\section{B. Estimation of kinetic constant and its variation range}

In Fig. 4, values of kinetic parameters, normalized to the true value, are presented for the case i) (only statistical uncertainties were took into account) and for the case ii) (systematic deviations were included in the fitting procedure), in both cases the variation range has been assessed from the simple and in principle inadequate (because systematic deviations are either not included or not normally distributed) $\chi^{2}$-test.

It can be seen that a poor determination of the kinetic parameters is achieved for case i), while method ii) does a much better job. Incidentally, it should be noted that for the case of method ii) and 3D-OSEM reconstructions, the estimation of the parameters includes in all cases within the error band the true values. In the case of 2D-OSEM and FBP, the true value does not lie in some cases, by a small margin, within the error band.
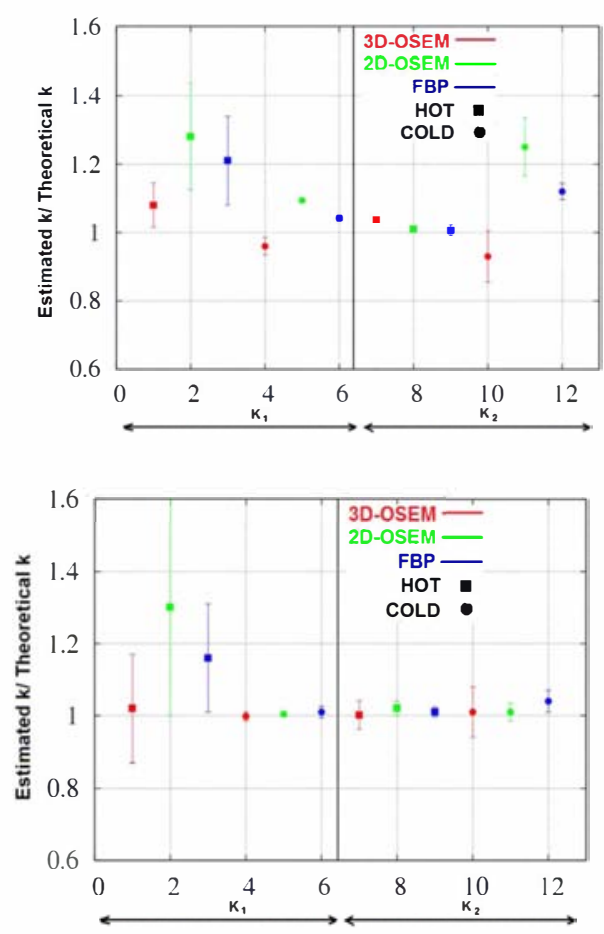

Fig.4. Kinetic parameters normalized to the true value obtained derived minimizing Eq. (3) with errors given by case i) (top) and ii) (bottom). The variation range has been assessed from $\chi^{2}$-test. $\mathrm{k}_{1}$ values (on the left side) and $k_{2}$ values (on the right side) are presented for the hot (square points) and cold (round points) regions
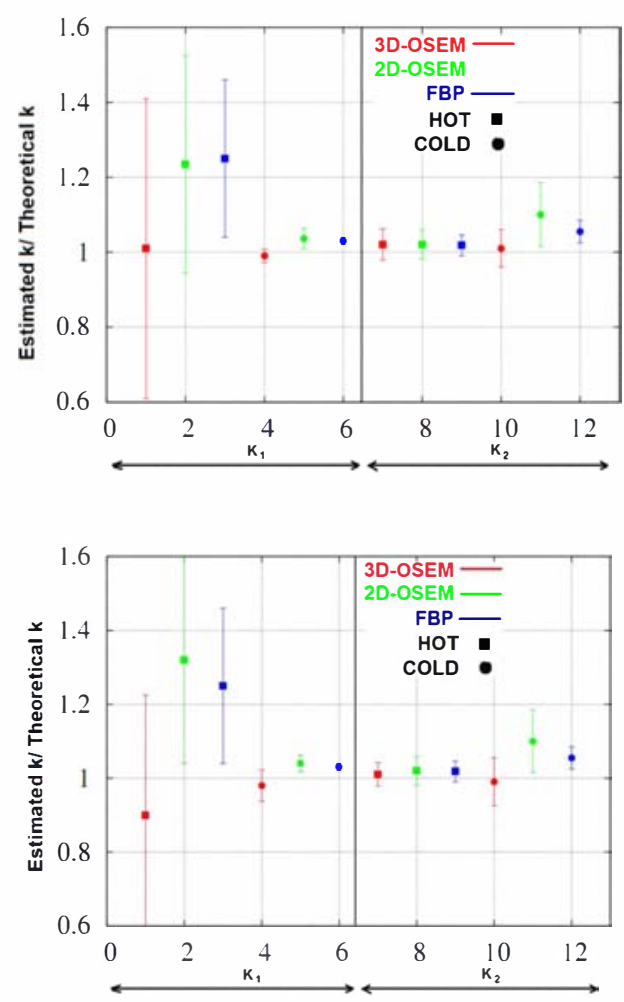

Fig.5. Kinetic parameters values normalized to the true value obtained from the three reconstructions methods employed for case i) (top) and ii) (bottom). The variation range has been assessed from pseudodata analysis. $\mathrm{k}_{1}$ values (on the left side) and $\mathrm{k}_{2}$ values (on the right side) are presented for hot (square points) and cold (round points) regions.

\section{DISCUSSION}

According to the results in these figures, we observe that, when the $\chi^{2}$-test from tabulated values is employed to estimate the ellipse errors and the variation range of the kinetic parameters (method 1): a) the constants derived from FBP images had less uncertainty when methods i) and ii) were used, but in some cases the deduced values did not agree with the real ones, within the error bands. b) For all reconstructions methods, the values derived from the fit are closer to the real ones when the systematic errors are employed in the fitting minimization. c) In the case of method ii), the 3D-OSEM algorithm is the one that yields kinetics parameters closer to the real ones, taking into account the error bands. d) The pseudodata analysis golden standard gives similar results with both methods i) and ii) and in both cases the parameters derived from the 3D-OSEM images included the true values within the likely range of variation. The pseudodata analysis can be easily implemented and it takes just a few minutes in an ordinary personal computer. Further, the results of the simple $\chi^{2}$-test for case ii), that is, taking into account systematic deviations in the denominator of Eq. (3) are very similar to the ones given by golden standard.

In summary, we have investigated the effect of the reconstruction method and fitting procedure on dynamic PET kinetic parameter estimation bias and reliability. We have found that if the observed bias in iterative methods is taken 
into account in the fitting procedure, it is possible to determine accurate kinetic parameters from these reconstruction methods. With this procedure 3D-iterative algorithms provide results which are in as good or better agreement with the theoretical values than those obtained from FBP reconstructed images. Also, when systematic errors are taken into account, the estimation of the variation range of kinetic constants obtained from the $\chi^{2}$-test, from tabulated values, is in good agreement with the results obtained from pseudodata analysis of data sets that model the systematic uncertainties.

A generalization of these results to other kinetic models and phantoms is under progress.

\section{REFERENCES}

[1] Tracer Kinetic Modeling in PET, chapter reproduced from Valk PE, Bailey DL, Towsend Dw, Misey MN. Dw, Misey MN. London Ltd 2003, 147-179

[2] Boellaard R, et al., "Experimental and clinical evaluation of iterative reconstruction (OSEM) in dynamic PET: Quantitative characteristics and effects on kinetic modeling", J. Nucl. Med. 42: 808-17, 2001.

[3] A. Reilhac, et al, "Simulation-based evaluation of OSEM iterative reconstruction methods in dynamic brain PET studies", NeuroImage 39: 359-368, 2008

[4] S España, et al., "PeneloPET, a Monte Carlo PET simulation tool based on PENELOPE: features and validation", Phys. Med. Biol. 54, 2009.

[5] Wang Y, et al., "Perfomance Evaluation of the GE Healthcare eXplore VISTA Dual-Ring Small-Animal PET Scanner". J. Nucl. Med. 47, 2006

[6] J.L. Herraiz et al., "FIRST: Fast Iterative Reconstruction Software for (PET) Tomography". Phys. Med. Biol. 51: 4547-4565, 2006.

[7] Statistical Data Analysis, Glen Cowan. Clarendon Press-Oxford, 1998. 